\title{
PROBING THE INTERSTELLAR MAGNETIC FIELD BY POLARIZATION OBSERVATIONS OF SUPERNOVA REMNANTS
}

\author{
Ernst FURST and Wolfgang REICH \\ Max-Planck-Institut für Radioastronomie \\ Auf dem Hügel 69 \\ 5300 Bonn 1, FRG
}

ABSTRACT. We present a comparison of the Galactic magnetic field and the magnetic field of three supernova remnants (SNRs) of bilateral symmetry derived from linear polarization measurements. These data are best explained if a quasi-perpendicular propagation of the SNR-shock wave is assumed.

Recently, Leckband et al. [1] studied the relation between the morphological appearance of SNRs and the Galactic magnetic field as modelled by Sofue and Fujimoto [2]. An improvement of this study is provided by taking into account the magnetic field structure of SNRs derived from polarization measurements at radio wavelengths. We have started an investigation of SNRs with bilateral symmetry, the morphology of which is supposed to be significantly influenced by the interstellar magnetic field local to the SNR. For a small sample of three SNRs of this type unambigous projected magnetic fields have been obtained. They are displayed
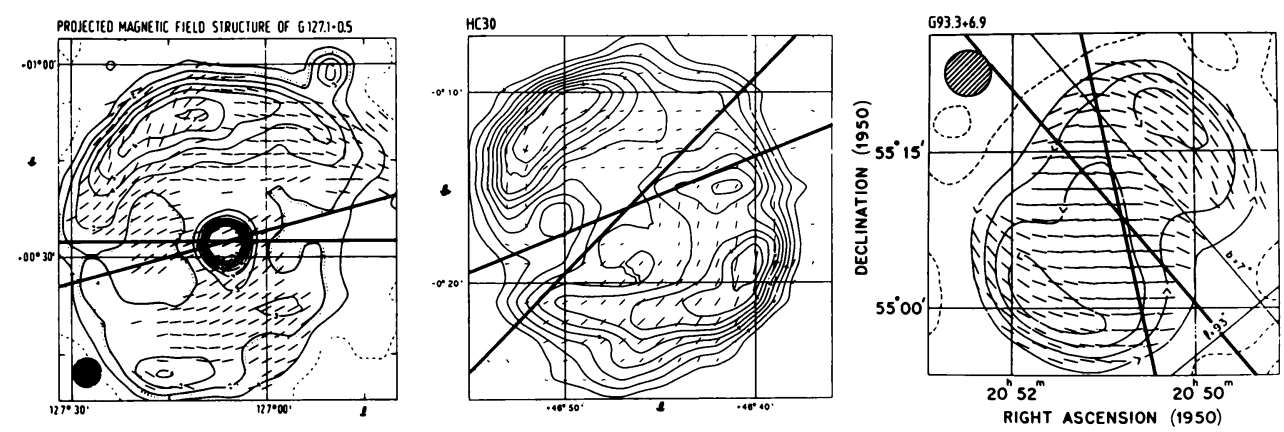

Figure 1. Three SNRs with bilateral symmetry. Contours of total intensity are shown with bars of the projected magnetic field direction superposed. Thick straight lines denote the Galactic magnetic field observed from starlight polarization [3]. The map of G127.1+0.5 has been taken from Fürst et al. [4] and the map of G93.3+6.9 is from Lalitha et al. [5]. The $10.7 \mathrm{GHz}$ map of $\mathrm{HC} 30$ is based on unpublished observations with the Effelsberg 100-m telescope.

R. Beck et al. (eds.), Galactic and Intergalactic Magnetic Fields, 73-75. (c) 1990 IAU. Printed in the Netherlands. 


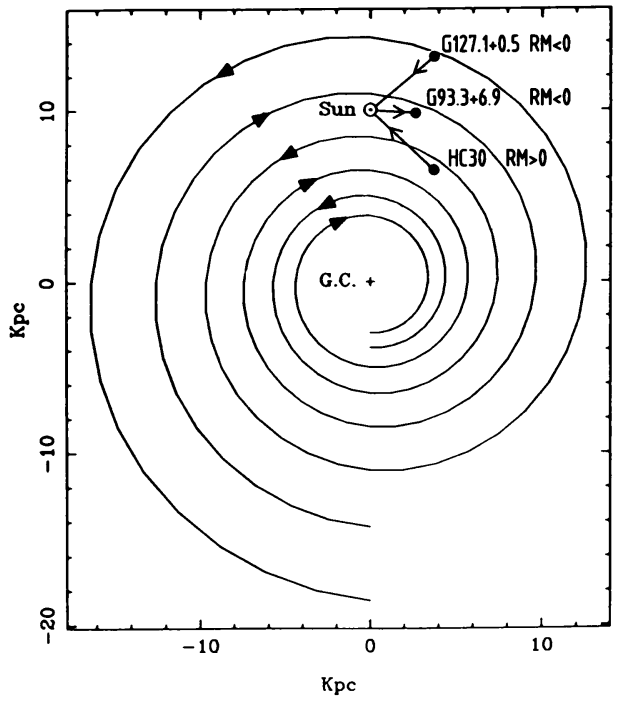

Figure 2. The Galactic magnetic field as modelled by sofue and Fujimoto [2] and the sign of rotation measure (RM) for the three SNRs as shown in Fig. 1.

in Fig. 1. For comparison the direction of the Galactic magnetic field as derived from linear polarization measurements of stars [3] nearest to the SNRs is shown in addition. Although the scatter is large, the Galactic magnetic field nicely fits the morphology and magnetic field structure of the SNRs, if quasi-perpendicular propagation of the SNR shock wave is assumed. We also compared the sign of Faraday rotation measure with the modelled Galactic magnetic field [2] (Figure 2) and found an agreement for the two SNRs HC30 and G93.3+6.9 and a disagreement for G127.1+0.5. However, deviations from the general Galactic magnetic field pattern will influence the sign of the rotation measure predominantly at large angles between the line of sight and the magnetic field direction.

At present we are going to observe a larger sample of suitable SNRs to confirm our preliminary conclusion:

1. The orientation of SNRs with bilateral symmetry depends on the Galactic magnetic field local to the SNR.

2. The bilateral symmetry in the radio emission from SNRs is caused by quasi-perpendicular propagation of the SNR shock wave.

3 . The Galactic magnetic field is at some places indeed not aligned with the Galactic plane, however, it is quasi-homogeneous over large distances.

\section{REFERENCES}

[1] Leckband, J.A., Spangler, S.R. and Cairns, I.H. (1989) ApJ. 338, 963

[2] Sofue, Y. and Fujimoto, M. (1983) ApJ. 265, 722

[3] Mathewson, D.S. and Ford, V.L. (1971) Mem. R. A. S. 74, 139

[4] Fürst, E., Reich, W. and Steube, R. (1984) Astron. Astrophys. 113, 11

[5] Lalitha, P., Mantovani, F., Salter, C.J. and Tomasi, P. (1984) Astron. Astrophys. 131, 196 
RUZMAIKIN: You said that the degree of polarization is zero in the case of a random magnetic field. However, the number of turbulent cells in SNR, as you have shown, does not exceed five. For such few cells one may expect a noticeable degree of polarization even for the random field. Did you take this point into account?

MILNE: What you say is true, now that we are clearly resolving the cells the polarization should be close to the $p_{\max }$ value, e.g. $\sim 70 \%$. However, the value is still only $\sim 5-10 \%$, perhaps suggesting that there is turbulence on a scale size much less than the cell size. This can be seen in the maps of Tycho's SNR (Dickel, van Breugel and Strom, this volume) and the Crab nebula (Bietenholz and Kronberg, this volume).

VOLK: Between the 2 classes of fields in SNRs - radial vs. tangential is there an evolutionary difference? There should be one.

MILNE: I guess I didn't really get that across in my talk. Yes, evolution is indicated in that all of the young SNRs exhibit radial fields as expected if they are dominated by the Rayleigh-Taylor instabilities. In contrast the older remnants are rather tangled, but some do show the tangential fields expected if they have reached the van der Laan compression regime. This can be seen in my "Atlas of SNR Magnetic Fields" (1987, Australian J. Phys. 40, 771).

DOGIEL: You have discovered the correlation between the large-scale magnetic field direction and an efficiency of the $C R$ acceleration in the SN envelopes. It seems that in this case there must be different spectra of accelerated electrons in different parts of the envelopes. Have you observed spectral index variations of the $\mathrm{SN}$ radio emission?

FURST: No spectral index variations in SNRs with bilateral symmetry have been found beyond $\Delta \alpha=0.2$, except in G127.1+0.5 where a small difference was found between the two halves.

VOLK: As far as I know diffusive acceleration in a perpendicular shock is possibly faster than in a parallel shock. Thus the maximum energy $\mathrm{E}_{\max }$ is higher, but not the particle intensity at an energy $E * E_{\max }$ as for radio synchrotron emission. Intensity variations could of course be due to variations of particle injection with shock angle. But this is difficult to estimate.

FURST: I reported here on an observational result which can best be explained by quasi-perpendicular shock propagation. If this result turns out to be true after confirmation by further observations of a larger sample, it may have implications for theoretical models of particle acceleration (or particle injection). 